\title{
Antiferromagnetic coupling in perpendicularly magnetized cubic and tetragonal Heusler bilayers
}

\author{
R. Ranjbar ${ }^{\mathrm{a}}$, K. Suzuki ${ }^{\mathrm{a}}$, A. Sugihara ${ }^{\mathrm{a}}$, Q. L. Ma ${ }^{\mathrm{a}}$, X. M. Zhang ${ }^{\mathrm{a}}$, T. \\ Miyazaki $^{\mathrm{a}}$, Y. Ando ${ }^{\mathrm{b}}$, S. Mizukami ${ }^{\mathrm{a}}$ \\ ${ }^{a}$ WPI Advanced Institute for Materials Research, Tohoku University, Sendai, Japan \\ ${ }^{b}$ Department of Applied Physics, Tohoku University, Sendai, Japan
}

\begin{abstract}
Epitaxial bilayer of tetragonal Heusler-like $D 0_{22}$-MnGa and cubic Heusler $L 2_{1^{-}}$$\mathrm{Co}_{2} \mathrm{MnSi}$ have been successfully grown even for an unannealed sample. The interfacial exchange coupling (Jex) for samples annealed at different temperatures was evaluated from the magnetic hysteresis loop. Antiferromagnetic Jex was observed in the interfaces of cubic Heusler $\mathrm{Co}_{2} \mathrm{MnSi}$ and tetragonal $\mathrm{DO}_{22^{-}}$ MnGa layers. The evaluated Jex is $-3.2 \mathrm{erg} / \mathrm{cm}^{2}$ for a bilayer film annealed at $400^{\circ} \mathrm{C}$, being comparable to that for the $\mathrm{FeCo} / \mathrm{MnGa}$ interface.
\end{abstract}

Keywords: $\mathrm{MnGa}$ film, $\mathrm{Co}_{2} \mathrm{MnSi}$ film, interfacial exchange coupling

2010 MSC: 00-01, 99-00

\section{Introduction}

To reduce switching current density while maintaining good thermal stability in spintronics applications like spin-transfer-torque magnetoresistive random access memory (STT-MRAM) based on magnetic tunnel junctions (MTJs), perpendicular magnetic anisotropy (PMA) films with low saturation magnetization $(M s)$, low damping constant $(\alpha)$, and high spin polarization $(P)$ are essential 1]. An excellent candidate for satisfying these properties is MnGa alloys [2, 3]. The structural, magnetic, and transport properties as well as spin polarization and magnetization dynamics of MnGa with PMA have been investigated in previous studies 4 9]. The new challenge concerning to MnGa

Email address: reza_ranjbar83@Yahoo.com (R. Ranjbar) 
alloys is increasing the tunnel magnetoresistance (TMR) ratio based on $\mathrm{MgO}-$ MTJs. One effective way is to insert an ultra-thin ferromagnetic metal/alloy interlayer such as Fe [7], $\mathrm{Co}$, and $\mathrm{FeCo}$ [9] between the $\mathrm{MnGa}$ and $\mathrm{MgO}$ barrier. The key in this kind of research is the Jex between MnGa alloy and insertion layer. Interestingly, the magnetic Jex was found to be antiferromagnetic (AF) for a Co-rich interlayer, when FeCo alloy interlayer was introduced [9]. AF coupling is very rare in magnetic films with PMA and is technologically important for achieving synthetic ferrimagnetic structure for STT-MRAM. The $\mathrm{FeCo} / \mathrm{MnGa}$ bilayer was used in making perpendicular MTJs based on MnGa film which showed largest TMR ratio up to $60 \%$ at room temperature. For further increase of TMR ratio, half-metallic Heusler $\mathrm{Co}_{2} \mathrm{MnSi} / \mathrm{MnGa}$ bilayer may be interesting because $\mathrm{Co}_{2} \mathrm{MnSi}(\mathrm{CMS})$ shows huge TMR ratio more than $100 \%$ even at room temperature [10,11]. However, there are no reports on such bilayer and also magnetic coupling of CMS and MnGa, which is fundamentally important to obtain perpendicular magnetization of CMS. We recently reported that $\mathrm{MnGa}$ film with interlayers of $\mathrm{B} 2$-ordered $\mathrm{Co}_{2} \mathrm{FeSi}(\mathrm{Al})$ cubic Heusler alloys exhibits AF (ferromagnetic and AF) Jex 12]. Therefore, it is interesting to study MnGa film with a CMS interlayer.

\section{Experimental}

CMS(20 nm) $/ D 0_{22}-\mathrm{MnGa}(30 \mathrm{~nm})$ bilayer films were epitaxially grown by using an ultrahigh vacuum magnetron sputtering system onto a $\mathrm{MgO}$ (100) substrate buffered and capped by a 10 and 5-nm-thick Cr layer, respectively. In-situ annealing was employed at $400^{\circ} \mathrm{C}$ after MnGa deposition. Ex-situ annealing was done at different annealing temperature $\left(T_{a}\right)$ at 200, 250,300,350, 400 , and $450^{\circ} \mathrm{C}$ by using a rapid thermal annealing (RTA) system. In addition, the 30 (20)-nm-thick Mn-Ga (CMS) films with the same stacking structure without CMS (MnGa) films were fabricated as reference films. The film compositions of MnGa and CMS were $\mathrm{Mn}_{70} \mathrm{Ga}_{30}$ and $\mathrm{Co}_{45} \mathrm{Mn}_{25} \mathrm{Si}_{30}$, respectively, as estimated by using inductively coupled plasma spectroscopy. For characterization of structural and magnetic properties, X-ray diffractometer with $\mathrm{Cu} K \alpha$ radiation, high-resolution transmission electron microscopy (HRTEM) 

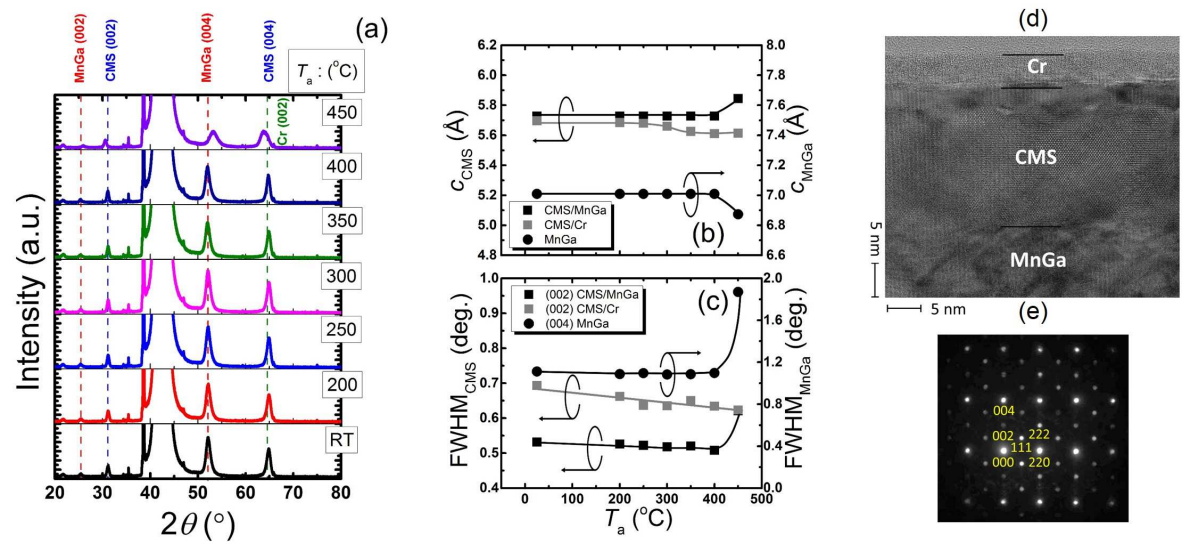

Figure 1: (a) XRD pattern of CMS/MnGa bilayers for unannealed sample and annealed samples at $200,250,300,350,400$, and $450^{\circ} \mathrm{C}$. (b) $T_{a}$ dependence of the $c$ lattice constant of MnGa, CMS/MnGa and CMS/Cr films. (c) $T_{a}$ dependence of the FWHM of MnGa, CMS/MnGa, and CMS/Cr films. (d) Cross-sectional HRTEM image and (e) nanobeam electron diffraction of a CMS film (with spot size $=1 \mathrm{~nm}$ ) annealed at $400^{\circ} \mathrm{C}$.

measurement, and vibrating sample magnetometer (VSM) were used.

\section{Results and discussion}

The out-of-plane X-ray diffraction (XRD) patterns of CMS/MnGa bilayers for different $T_{a}$ are shown in Fig. 1(a). The peaks of MnGa (002) and (004) and CMS (002) corresponding to $D 0_{22}$ and $L 2_{1}$ structures of MnGa and CMS are clearly observed even for an unannealed sample indicating good epitaxy of CMS on MnGa film. In addition to these peaks, we observed a (004) peak of the CMS film that overlapped the Cr peak. The $T_{a}$ dependence of the $c$ lattice constant of MnGa and CMS bilayer films (CMS/MnGa) and also of CMS reference films (CMS/Cr) are shown in Fig. 1(b). The $c$ lattice constant of $\mathrm{MnGa}$ is constant and very close to $7.00 \AA$, the value of the reference $\mathrm{MnGa}$ film for the unannealed sample and samples annealed at $200^{\circ} \mathrm{C}$ up to $400^{\circ} \mathrm{C}$. The $c$ lattice constant of MnGa is drastically reduced after annealing at $450^{\circ} \mathrm{C}$. For the $c$ lattice constant of the CMS bilayer films the opposite behaviors were observed. These changes are attributed to interdiffusion in the high $T_{a}$. The $c$ 
lattice constant of CMS reference films decreases after annealing at $300^{\circ} \mathrm{C}$ and then becomes a constant value that is close to that of the bulk (5.65 $\AA$ ) owing to crystallization of CMS on Cr at high $T_{a}$. The difference between the $c$ lattice constant of the CMS bilayer and that of the reference films shows an expansion of the $c$ lattice constant in the out-of-plane direction for better matching of the CMS layer to the MnGa layer. The $a$ lattice constant of MnGa and CMS films are 3.937 and $5.567 \AA$ for the unannealed CMS/MnGa bilayer, respectively estimated from in-plane XRD measurement. The estimated mismatch value $(0.013 \%)$ is smaller than bulk values $(2.17 \%)$ which indicates the lattice of CMS contracted to fit the MnGa lattice on the bottom in the CMS/MnGa bilayer.

The $T_{a}$ dependence of the full width at half maximum (FWHM) for the MnGa, CMS/MnGa, and CMS/Cr peaks is shown in Fig. 1(c). The FWHM for CMS/Cr decreases linearly which indicates the improving of quality of the film at high $T_{a}$ because of crystallization. The same behaviors were observed for MnGa and CMS/MnGa films, but the FWHM drastically increases above $400^{\circ} \mathrm{C}$ because interdiffusion. The smaller FWHM for CMS/MnGa compared to CMS/Cr indicates that the quality of the CMS film on the MnGa layer is better than that of the CMS film on the Cr layer. A cross-sectional HRTEM image and nanobeam electron diffraction of the sample annealed at $400^{\circ} \mathrm{C}$ are shown in Figs. 1(d) and (e), respectively. This image clearly shows that all the layers of the CMS/MnGa bilayer were grown epitaxially and were single crystalline. Furthermore, nanobeam electron diffraction patterns for CMS film showed a (111) superlattice spot, indicating an $L 2_{1}$ ordered structure corresponding to the XRD result. Typical out-of-plane and in-plane $M-H$ loops measured by using a VSM for the unannealed CMS/MnGa bilayer are shown in Fig. 2(a). The 30-nm-thick MnGa and 20-nm-thick CMS films showed hard and soft magnetic properties, respectively (the data for which are not shown here). As can be seen from Fig. 2(a), the magnetization of the CMS/MnGa bilayer film appears to be a combination of magnetization of MnGa and CMS films with the Jex at the interface of MnGa and CMS films taken into account. We marked three magnetic fields with $H_{+}, H_{-}$, and $H_{S W}$ corresponding to the saturation field in the parallel and antiparallel states and the switching field, 


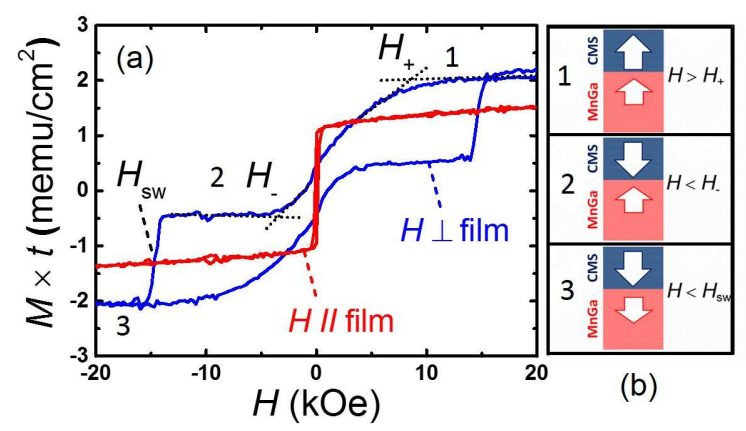

Figure 2: (a) Typical VSM $M-H$ loop for the unannealed CMS/MnGa bilayer film. (b) Magnetization configurations of CMS and MnGa films corresponding to numbers 1, 2, and 3.

respectively. In addition, the magnetization configurations of CMS and $\mathrm{MnGa}$ films are shown in Fig. 2(b). The magnetization of MnGa and CMS are parallel for $H>H_{+}$in step 1, as shown in Fig. 2(b). With decreasing magnetic field, the magnetization of CMS starts to rotate from parallel to become antiparallel by step 2. Then the magnetization of MnGa switches from the up direction to the down direction with further increase of the absolute value of the magnetic field in step 3. Taking into account AF Jex at the interface, we would expect different values of $H_{+}$and $H_{-}$. By increasing the coupling strength, $H_{+}$ increases whereas the absolute value of $H_{-}$decreases.

Fig. 3(a) shows the $T_{a}$ dependence of saturation magnetization which is estimated from the out-of-plane $M-H$ loop for the CMS/MnGa and CMS/Cr films. For the unannealed CMS/Cr film, the magnetization is too small. With increasing $T_{a}$, the magnetization of CMS increases slowly and then drastically increases above $300^{\circ} \mathrm{C}$. Finally, the magnetization attains its maximum value for samples annealed at 400 and $450^{\circ} \mathrm{C}$ because of crystallization of the CMS film. In contrast, the magnetization of the CMS film on the MnGa layer increases slightly with increasing $T_{a}$ and then decreases drastically after annealing at $400^{\circ} \mathrm{C}$ because of the effect of interdiffusion. Such behaviors are consistent with structure changes, as can be seen from the XRD result. The larger magnetization of the CMS film on the MnGa layer, especially for the unannealed sample, compared with the magnetization of the CMS film on the 

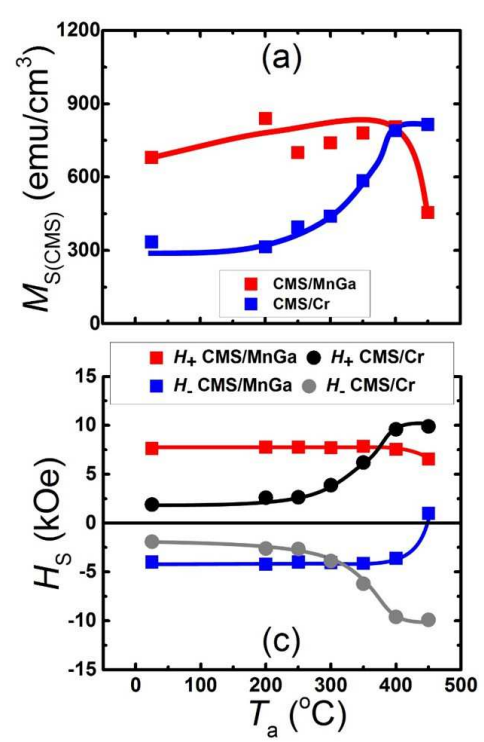
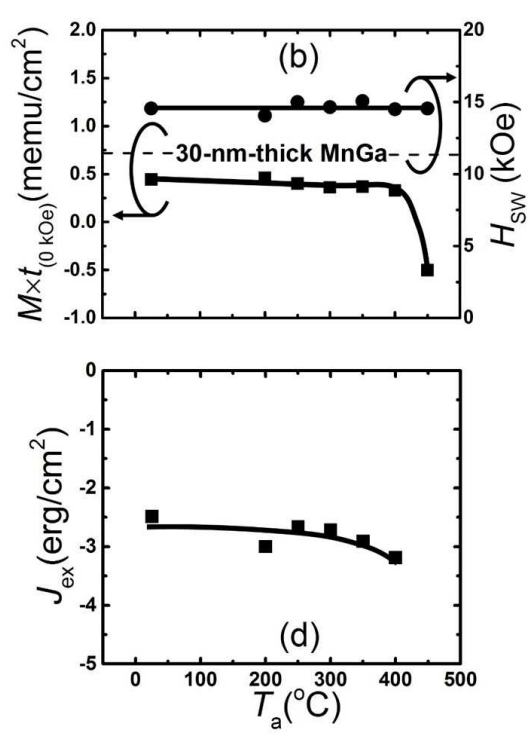

Figure 3: (a) $T_{a}$ dependence of saturation magnetization for CMS films on MnGa and Cr layers. (b) $T_{a}$ dependence of remnant magnetization, switching field, and (c) saturation fields $\left(H_{+}\right.$and $\left.H_{-}\right)(\mathrm{d})$ as well as Jex constant for CMS (20 nm)/MnGa (30 nm) bilayer films.

Cr layer suggests better crystallization of the CMS film on the MnGa layer. The value of magnetization is comparable with those from other reports [13, 14].

The $T_{a}$ dependence of the remanent magnetization $\left(M \times t_{(0 k O e)}\right)$ and $H_{S W}$ is shown in Fig. 3(b). When a hard magnetic film combines with a soft magnetic film in the case of no coupling $H_{S W}$ and $M \times t_{(0 k O e)}$ of the bilayer are the same as those for the hard magnetic film, whereas in the case of AF coupling larger $H_{S W}$ and smaller $M \times t_{(0 k O e)}$ are expected $[15]$. As can be seen from Fig. 3(b), values for $M \times t_{(0 k O e)}$ and $H_{S W}$ of CMS/MnGa bilayer films are smaller and larger than those of 30-nm-thick MnGa film, respectively. The $T_{a}$ dependence of $H_{+}$and $H_{-}$for CMS films on MnGa and Cr layers is shown in Fig. 3(c). The $H_{+}$and $H_{-}$for CMS films on $\mathrm{Cr}$ layers show symmetry, as we expect for the no-coupling case, whereas for the CMS films on MnGa asymmetry was observed as a result of AF Jex between MnGa and CMS films. The $H_{+}$and $H_{-}$values for a CMS film on a MnGa layer did not change as $T_{a}$ no higher than $400^{\circ} \mathrm{C}$.

Fig. 3(d) shows the $T_{a}$ dependence of the Jex for CMS/MnGa bilayer films. 
We evaluated $J_{e x}$ by using the following equation:

$$
H_{ \pm}= \pm 4 \pi M_{\mathrm{eff}}^{\mathrm{CMS}}-\left(J_{\mathrm{ex}} / M_{\mathrm{S}}^{\mathrm{CMS}} d_{\mathrm{CMS}}\right)
$$

where $M_{S}^{\mathrm{CMS}}$ and $d_{C M S}$ are the saturation magnetization and thickness of the CMS film, respectively. The $J_{e x}$ value for the unannealed CMS/MnGa bilayer film is $-2.5 \mathrm{erg} / \mathrm{cm}^{2}$ and becomes stronger with increasing annealing temperature, and reaching $-3.2 \mathrm{erg} / \mathrm{cm}^{2}$ for the sample annealed at $400^{\circ} \mathrm{C}$. These changes are attributed to improvement of interfacial quality of CMS and MnGa films because of annealing as can be seen from XRD result. The absolute values of negative $J_{e x}$ are comparable to those evaluated in the $\mathrm{FeCo} / \mathrm{MnGa}$ bilayers [9] and larger than those in $\mathrm{Co}_{2} \mathrm{FeSi}(\mathrm{Al}) / \mathrm{MnGa}$ [12].

\section{Conclusions}

In summary, the $T_{a}$ dependence on the structural and magnetic properties of CMS/MnGa bilayer films has been investigated. Bilayer films have been successfully grown even for unannealed sample. XRD measurement results show the better epitaxy of CMS film on MnGa compared with Cr. Nanobeam electron diffraction patterns for CMS film showed a (111) superlattice spot, indicating an $L 2_{1}$ ordered structure after annealing at $400^{\circ} \mathrm{C}$. $\mathrm{AF}$ Jex was observed for CMS/MnGa bilayer film. The coupling is stronger than that of $\mathrm{Co}_{2} \mathrm{FeSi}(\mathrm{Al}) / \mathrm{MnGa}$ and comparable with those evaluated in the $\mathrm{FeCo} / \mathrm{MnGa}$ bilayer film.

\section{Acknowledgment}

This work was partially supported by ASPIMATT (JST), Asahi Glass Foundation and a Grant-in-Aid for Scientific Research (No. 24686001). R.R also thanks Y. Kondo for his technical assistance and the WPI program and acknowledges a JGC-S scholarship (Nikki).

\section{References}

[1] H. Yoda, et al., Curr. Appl. Phys. 10 (2010) e87. 
[2] F. Wu, S. Mizukami, D. Watanabe, H. Naganuma, M. Oogane, Y. Ando, and T. Miyazaki, Appl. Phys. Lett. 94 (2009) 122503.

[3] B. Balke, G.H. Fecher, J. Winterlik, and C. Felser, Appl. Phys. Lett. 90 (2007) 152504.

[4] K. Wang, E. Lu, J.W. Knepper, F. Yang, and A.R. Smith, Appl. Phys. Lett. 98 (2011) 162507.

[5] H. Kurt, K. Rode, M. Venkatesan, P. Stamenov, and J.M.D. Coey, Phys. Rev. B. 83 (2011) 020405.

[6] A. Köhler, I. Knez, D. Ebke, C. Felser, and S. Parkin, Appl. Phys. Lett. 103 (2013) 162406.

[7] T. Kubota, Q.L. Ma, S. Mizukami, X.M Zhang, H. Naganuma, M. Oogane, Y. Ando, and T. Miyazaki,J. Phys. D. 46 (2013) 155001.

[8] R. Ranjbar, S. Mizukami, Y. Ando, T. Kubota, Q.L. Ma, X.M Zhang, and T. Miyazaki, J. Magn. Magn. Mater. 346 (2013) 53

[9] Q.L. Ma, S. Mizukami, T. Kubota, X.M. Zhang, Y. Ando, and T. Miyazaki, Phys. Rev. Lett. 112 (2014) 157202.

[10] Y. Sakuraba, M. Hattori, M. Oogane, Y. Ando, H. Kato, A. Sakuma, and T. Miyazaki, Appl. Phys. Lett. 88 (2006) 192508.

[11] H. Liu, Y. Honda, T. Taira, K. Matsuda, M. Arita, T. Uemura, and M. Yamamoto, Appl. Phys. Lett. 101 (2012) 132418.

[12] R. Ranjbar, K. Suzuki, A. Sugihara, Q. L. Ma, X. M. Zhang, T. Miyzaki, Y. Ando, and S. Mizukami, J. Appl. Phys. 117 (2015) 17A332.

[13] S. Tsunegi, Y. Sakuraba, M. Oogane, K. Takanashi, and Y. Ando, Appl. Phys. Lett. 93 (2008) 112506.

[14] Y. Sakuraba, et al., Phys. Rev. B 82 (2010) 094444.

[15] G. Asti, M. Ghidini, R. Pellicelli, C. Pernechele, M. Solzi,F. Albertini, F. Casoli, S. Fabbrici, and L. Pareti, Phys. Rev. B. 73 (2006) 094406. 